\title{
Mediterranean diet in secondary prevention of CHD
}

\author{
Michel de Lorgeril* and Patricia Salen \\ Laboratoire Cœur and Nutrition, TIMC-IMAG, CNRS UMR 5525, Faculté de Médecine de Grenoble, Université \\ de Grenoble, 38706 La Tronche, France
}

Submitted 13 May 2011: Accepted 12 August 2011

\begin{abstract}
Objective: To summarise our present knowledge on the Mediterranean diet in secondary prevention of CHD.

Design: Review of literature.

Setting: Adult coronary patients.

Subjects: CHD patients at high risk of cardiac death.

Results: The two main causes of death in these patients are sudden cardiac death (SCD) and chronic heart failure (CHF). The main mechanism underlying recurrent cardiac events is coronary thrombosis resulting from atherosclerotic plaque erosion or ulceration. The occurrence of thrombosis is usually associated with plaque weakness in relation to high lipid content of the lesion where cholesterol only represents a very small part compared with other lipids (i.e. fatty acids). Thus, the three main aims of the preventive strategy are to prevent coronary thrombosis, malignant ventricular arrhythmia and the development of left ventricular dysfunction (and CHF) and finally to minimise the risk of plaque erosion and ulceration. There is now a consensus about recommending the Mediterranean diet pattern for the secondary prevention of CHD because no other dietary pattern has been successfully tested so far in these patients. The most important aspect, in contrast with the pharmacological prevention of CHD (including cholesterol lowering), is that the Mediterranean diet results in a striking effect on survival.

Conclusions: The traditional Mediterranean diet is effective in reducing both coronary atherosclerosis/thrombosis and the risk of fatal complications such as SCD and heart failure.
\end{abstract}

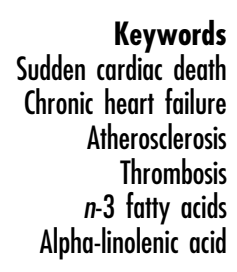

The priority of secondary prevention is somewhat different from that of primary prevention. In the context of primary prevention, intervention focuses on traditional risk factors (e.g. lifestyle factors, diabetes, overweight or obesity, high blood pressure) and surrogate endpoints. In secondary prevention, it is critical to reduce the risk of severe and often fatal clinical complications such as sudden cardiac death (SCD) and chronic heart failure $(\mathrm{CHF})^{(1,2)}$. This does not mean that traditional risk factors of $\mathrm{CHD}$ should not be measured and, if necessary, corrected in secondary prevention, because they also play a role in the occurrence of CHD complications. It simply means that because complications such as SCD and associated syndromes are often unpredictable, occur out of hospital and far from any potential therapeutic resources in the majority of cases, and account for approximately $70 \%$ of cardiac mortality in secondary prevention, they should be the priority of any secondary prevention programme. For this reason, in the present text, we will focus our recommendations and comments specifically on clinical efficacy and not on surrogate efficacy ${ }^{(1,2)}$.

Whatever the specific clinical aims of the programme, nutritional evaluation and counselling of each individual with CHD must be a key goal of the preventive intervention.
Nutrition is, however, only one component of such a programme. Exercise training, behavioural interventions (particularly to help the patient abstain from smoking) and drug therapy - particularly antithrombotic drugs also have important roles. The dietary prevention programme is commonly initiated during hospitalisation for a first CHD event. With the shortening of stay in the coronary care unit, the dietary intervention is initiated during the following days in hospital, and then continued in secondary prevention centres and included in cardiac rehabilitation programmes. An individualised dietary prevention programme should be developed under the guidance of a specialised dietician and in close collaboration with the patient's cardiologist and primary care physician, so that there is no discontinuity or discrepancy in dietary counselling between the hospitalisation and post-hospitalisation phases of the rehabilitation programme ${ }^{(1,2)}$.

\section{The scientific concept of Mediterranean diet}

There is now a consensus about recommending the Mediterranean diet pattern for the secondary prevention 
of CHD because no other dietary pattern has been successfully tested so far in these patients ${ }^{(1,2)}$. The most important aspect, in contrast with the pharmacological prevention of CHD (including cholesterol lowering), is that the Mediterranean diet results in a striking effect on survival. The main explanation is that the Mediterranean diet is protective not only against CHD and CHD complications, but also against other chronic diseases including cancers $^{(3,4)}$. Furthermore, the Mediterranean diet appears to be effective in reducing both coronary atherosclerosis/ thrombosis and the risk of fatal complications (SCD and $\mathrm{CHF}$ ) of atherosclerosis and thrombosis.

Finally, no harmful side effects have been reported following the adoption of this dietary pattern, unlike drug therapies ${ }^{(4,5)}$.

Prospective studies of the epidemiology of CHD have shown that mortality from CHD differs greatly among populations and that at least some of the differences are associated with differences in dietary habits ${ }^{(6)}$. Mediterranean populations have been relatively protected from CHD and certain cancers, and the particular composition of the traditional Mediterranean diet has been put forth to explain this ${ }^{(7,8)}$. However, epidemiological studies only provide associations between the risk factors and clinical endpoints, not causal relationships. Several confounding factors may play a part in these associations. The economic situation and the presence of extended social support systems, for instance, have been proposed to explain the low prevalence of CHD in some Mediterranean countries. Clearly, randomised trials are the only way to make sure that a given dietary pattern results in a significant protective effect against CHD complications.

Some dietary trials in primary or secondary prevention of CHD have reported an impressive reduction of CHD risk, especially in terms of mortality ${ }^{(9-11)}$.

In contrast, other dietary trials specifically aimed at reducing blood cholesterol failed to significantly improve the prognosis of the dieters ${ }^{(12-14)}$.

The successful trials in general tested dietary patterns characterised by a low intake of total, saturated and $n-6$ polyunsaturated fats ${ }^{(9-11)}$ and an increased intake of $n-3$ fatty acids ${ }^{(9-11,15)}$. Their aim was not to primarily reduce blood cholesterol, but this is a critical issue.

Two of these trials ${ }^{(9,10)}$ also included a high intake of fresh fruits and vegetables, legumes and cereals containing large amounts of fibre, antioxidants, minerals, vegetable proteins and vitamins of the B group. The credibility of these trials was considerably reinforced by a number of studies showing protective effects of most of these foods and nutrients, ${ }^{(16-18)}$ with a particular emphasis on plant and marine $n-3$ fatty acids ${ }^{(5,10,11,15,19,20)}$.

The 'Lyon Diet Heart Study' was a randomised singleblind secondary prevention trial aimed at testing whether an experimental Mediterranean diet could reduce the risk of recurrence after a first myocardial infarction. A significant reduction of the rates of fatal and non-fatal cardiovascular complications was reported $^{(5,10,21)}$, and no major bias was detected in the trial ${ }^{(5,22)}$. In addition, the trial suggested for the first time that patients following the Mediterranean diet were also relatively protected from cancer ${ }^{(4)}$. Although further controlled trials are warranted to confirm the cancer data, those obtained from the 'Lyon Diet Heart Study' are in line with several epidemiological observational studies suggesting that some dietary factors are very important in cancers and cancer prevention ${ }^{(3,23-26)}$.

In the 'Lyon trial', investigators advised patients to use either olive oil or rapeseed oil (or both oils together) because some French patients do not like the taste of olive oil and might have rejected the whole Mediterranean diet pattern. As rapeseed and olive oil have similar fatty acid compositions - very low saturated fatty acids and high oleic acid - the tested diet remains, on average, a very Mediterranean diet ${ }^{5,10,21)}$.

In a recent and very large observational study about the health effects of the Mediterranean diet ${ }^{(27)}$, the authors concluded that their results provide strong evidence for a beneficial effect of higher conformity with the Mediterranean dietary pattern on risk of death from all causes, including deaths due to CVD and cancer, in a US population. They base their conclusions on previous epidemiological studies conducted in non-US populations and reporting similar data about the effect of the Mediterranean diet on longevity ${ }^{(3,28)}$.

Thus, epidemiological studies ${ }^{(3,27,28)}$ confirmed the results of the 'Lyon Diet Heart Study', now a reference trial in the field ${ }^{(29)}$. Interestingly, in that trial, there was no difference between groups for the main conventional risk factors, including blood cholesterol and blood pressure. This suggested that protection was largely independent from these traditional (conventional) factors.

In contrast, it is noteworthy that a recent meta-analysis involving 534906 individuals reported that the Mediterranean diet was associated with a significant reduction of metabolic syndrome, a pre-diabetes state ${ }^{(30)}$.

All these data are of considerable relevance for public health. No other dietary pattern is apparently as effective in reducing the risks of both diabetes and cardiovascular complications. It is therefore surprising that when defining dietary recommendations to prevent chronic diseases, experts forget to mention the Mediterranean diet ${ }^{(31)}$, sometimes distort the published data to discredit the concept, and put forward treatments that increase the risk of diabetes ${ }^{(32,33)}$.

\section{The Mediterranean diet in practical terms}

The diet score usually used to assess conformity with the Mediterranean dietary pattern in epidemiological studies $^{(3,27,28,30)}$ is rather simplistic and does not capture the various practical aspects of the real and various traditional Mediterranean diets.

Briefly, what must clinicians (and their patients) know? 
The Mediterranean $\operatorname{diet}^{(7,8)}$ is characterised by the consumption of:

1. A wide variety of raw, sometimes cooked, seasonal vegetables throughout the year, often large amounts of onions, garlic, parsley, rosemary, oregano, thyme and other aromatic herbs.

2. Fruit throughout the year, both fresh and dried (during the summer, for consumption in winter, e.g. apricots, figs and grapes).

3. Various nuts (almonds, hazelnut), particularly walnuts, which are rich in alpha-linolenic acid (ALA), the main plant $n-3$ and a major characteristic of traditional Mediterranean diets ${ }^{(8)}$. There are many other sources of ALA in Mediterranean diets, including salads such as purslane ${ }^{(34)}$ and products from animals fed with ALA-rich feed such as linseed (rabbit, eggs and chicken, dairy products).

4. Grains, preferably whole, especially wheat in the form of bread, fermented with natural leaven and sometimes flavoured with ALA-rich linseed. The wheat used in traditional Mediterranean diets (like the vegetables and fruit) is rustic and does not contain pesticides as it is not a product of industrial agriculture.

5. Fatty fish, including anchovy, sardine, mackerel, sea bream and red tuna, all rich in very-long chain (marine) $n-3$ fatty acids. Another source of indispensable marine $n-3$ fatty acids may be the eggs of linseed-fed chicken, as well as the 'fish-like effect of moderate wine drinking,(35).

6. Olive oil, the main edible oil used in the Mediterranean area, low saturated and rich monounsaturated. However, the monounsaturated fat/saturated fat ratio used by epidemiologists does not capture one major lipid characteristic of the Mediterranean diet, which is low in $n-6$ and rich in $n-3$ fatty acids. The $n-6 / n-3$ ratio has been proposed as a major component of a healthy $\operatorname{diet}^{(36)}$.

7. In contradiction with many experts, Mediterranean populations do traditionally eat dairy products, though made of goat and ewe milk and not cow milk. Importantly, these are consumed in the fermented forms of cheese and yoghurt, and almost never as milk, butter or cream.

8. Mediterranean populations are not vegetarian. They eat ALA-rich eggs and small amounts of meat, mainly lean meat such as rabbit, chicken and duck. Beef and/ or pork are also on the menu in the North of the area, while mutton is the preferred meat for festive meals in the South. It is also important to note that everywhere in the Mediterranean area the diet includes a large number of legumes and is therefore rich in vegetable proteins.

9. Moderate alcohol drinking, essentially during meals, is a major characteristic of the Mediterranean diet. The main alcoholic beverage is wine, particularly red wine, a major source of various polyphenols. Wine is a mix of ethanol and polyphenols. South of the Mediterranean Sea, the main source of healthy polyphenols is not wine but fermented black tea (a mix of water and polyphenols). Thus, most people living in the Mediterranean area are high consumers of various polyphenols whose health effects ${ }^{(37)}$ are still considerably underestimated by scientists and physicians. This is another major item not included in the Mediterranean diet score used by epidemiologists.

\section{The wine drinking issue}

The medical and scientific literature shows that moderate drinking (one to two drinks per day for women and two to four drinks per day for men) is usually associated with a better life expectancy in the general population, as well as in patients with established $\mathrm{CHD}^{(38-40)}$. In the absence of a controlled trial, which is neither technically nor ethically feasible, the main question for physicians remains whether the inverse association between moderate drinking and CHD complications is a cause-effect relationship.

Although prospective studies with light drinkers (rather than non-drinkers) as the referent group have shown that the 'sick quitter bias' is not the main explanation for the protective effect of moderate drinking, a recent study, in which former drinkers were examined separately from long-term abstainers, confirmed that protection is still present when only long-term abstainers are included in the referent group $^{(41)}$. This was an important finding because it strongly supports the cause-effect relationship between moderate drinking and better survival.

In meta-analyses, moderate drinking generally results in approximately $30 \%$ lower cardiac mortality and a $20 \%$ reduction of all-cause mortality, ${ }^{(38)}$ which is considerable when compared with the effect of drug treatment and in terms of public health. In addition to the strong epidemiological evidence (and in the absence of clinical trials), another way of evaluating the relationship between moderate drinking and survival is to examine the biological mechanisms by which moderate drinking may reduce the risk of cardiac death and improve survival. In addition to the well-known effects of alcohol on haemostasis (through reduced platelet function and fibrinogen levels) and insulin resistance, recent data indicate that moderate drinking may have a direct protective effect on the ischaemic myocardium ${ }^{(42)}$, and may positively interact with $n-3$ fatty acids, ${ }^{(35)}$ known to be highly protective in secondary prevention, especially against SCD ${ }^{(15)}$.

These two mechanisms are important to know because they may partly explain why moderate drinking was shown to reduce the risk of $\mathrm{SCD}^{(43)}$, a complication that accounts for $65-75 \%$ of all cardiac deaths in the US population ${ }^{(44)}$. Thus, epidemiological and biological studies strongly suggest that moderate drinking results in 
reduced mortality and better life expectancy in patients with established CHD.

Finally, regarding alcohol consumption in secondary prevention, it is important to:

1. identify those at very high risk of cardiac death, for whom there is a clear indication for an implantable cardiac defibrillator (ICD);

2. identify binge and heavy drinkers and explain to them a better way of drinking to protect their lives;

3. identify non-drinkers (and respect their choice), but also those who abstain because they wrongly believe that even light drinking is bad for their health;

4. explain to all patients (with or without ICD) that moderate drinking, especially (but not only) in the form of wine in the context of the traditional Mediterranean diet, may be the most effective way to prevent both fatal and nonfatal complications of $\mathrm{CHD}^{(8,39)}$, even in Northern Europe and in old age ${ }^{(45)}$. Further studies are needed to fully understand the mechanisms of this protection. Finally, coming back to the Mediterranean diet and to the specific consumption of wine, the next question is whether wine drinking (the preferred beverage of Mediterranean populations) is superior to other alcoholic beverages for the prevention of $\mathrm{CHD}$ complications. Recent observational data and metaanalysis suggest that wine is more protective than beer and spirits ${ }^{(46)}$.

\section{Conclusion}

The traditional Mediterranean diet is effective in reducing both coronary atherosclerosis/thrombosis and the risk of fatal complications such as SCD and CHF.

\section{Acknowledgements}

The authors declare that they have no conflict of interest.

\section{References}

1. de Lorgeril M \& Salen P (2003) Dietary intervention in coronary care unit and in secondary prevention. In Acute Coronary Syndromes: A Companion to Braunwald's Heart Disease, vol. 44, pp. 613-631 [P Theroux, editor]. Philadelphia, USA: Saunders.

2. de Lorgeril M \& Salen P (2007) Secondary prevention of coronary heart disease: impact of nutrition on the risk of fatal complications. In Cardiovascular prevention and rehabilitation, vol. 23, pp. 181-194 [C Monpère editor]. London, UK: Springer.

3. Trichopoulou A, Costacou T, Barnia C et al. (2003) Adherence to a Mediterranean diet and survival in a Greek population. N Engl J Med 348, 2599-2608.

4. de Lorgeril M, Salen P \& Martin JL (1998) Mediterranean dietary pattern in a randomized trial: prolonged survival and possible reduced cancer rate. Arch Intern Med 158, 1181-1187.

5. de Lorgeril M, Salen P \& Martin JL (1999) Mediterranean diet, traditional risk factors and the rate of cardiovascular complications after myocardial infarction. Final report of the Lyon Diet Heart Study. Circulation 99, 779-785.

6. Sans S, Kesteloot H \& Kromhout D (2006) Dynamics of cardiovascular and all-cause mortality in Western and Eastern Europe between 1970 and 2000. Eur Heart J 27, 107-113.

7. Willett WC, Sacks F \& Trichopoulou A (1995) Mediterranean diet pyramid: a cultural model for healthy eating. $\mathrm{Am}$ J Clin Nutr 61, Suppl. 6, 1402S-1406S.

8. de Lorgeril M \& Salen P (2007) Modified Cretan Mediterranean diet in the prevention of coronary heart disease and cancer: an update. World Rev Nutr Diet 97, 1-32.

9. Hjermann I, Holme I \& Leren P (1986) Oslo Study Diet and Antismoking Trial. Results after 102 months. Am J Med 80, 7-11.

10. de Lorgeril M, Renaud S \& Mamelle N (1994) Mediterranean alpha-linolenic acid-rich diet in secondary prevention of coronary heart disease. Lancet 343, 1454-1459.

11. Burr ML, Fehily AM \& Gilbert JF (1989) Effects of changes in fat, fish, and fibre intakes on death and myocardial reinfarction: diet and reinfarction trial. Lancet 334, 757-761.

12. de Lorgeril M, Salen P, Monjaud I et al. (1997) The diet heart hypothesis in secondary prevention of coronary heart disease. Eur Heart J 18, 14-18.

13. Ramsden CE, Hibbeln JR, Majchrzak SF et al. (2010) N-6 fatty acid-specific and mixed polyunsaturated dietary interventions have different effects on CHD risk: a meta-analysis of randomised controlled trials. Br J Nutr 104, 1586-1600.

14. Ramsden CE, Hibbeln JR, Majchrzak SF et al. (2011) Don't disregard the essential distinction between PUFA species. Br J Nutr 17, 1-5.

15. GISSI-Prevenzione Investigators (1999) Dietary supplementation with n-3 polyunsaturated fatty acids and vitamin $\mathrm{E}$ after myocardial infarction: results of the GISSI-Prevenzione trial. Lancet 354, 447-455.

16. Gilman MW, Cupples LA \& Gagnon D (1995) Protective effect of fruits and vegetables on development of stroke in men. JAMA 273, 1113-1117.

17. Rimm EB, Ascherio A \& Giovannucci E (1996) Vegetable, fruit and cereal fiber intake and risk of coronary heart disease among men. JAMA 275, 447-451.

18. Key TJ, Thorogood M, Appleby PN et al. (1996) Dietary habits and mortality in 11,000 vegetarians and health conscious people: results of a 17-year follow-up. BMJ 313, 775-779.

19. Albert CM, Hennekens CH \& O'Donnell CJ (1998) Fish consumption and risk of sudden death. JAMA 279, 23-28.

20. Daviglus ML, Stamler J \& Orencia AJ (1997) Fish consumption and the 30-year risk of fatal myocardial infarction. $N$ Engl J Med 336, 1046-1053.

21. de Lorgeril M, Salen P \& Martin JL (1996) Effect of a Mediterranean-type of diet on the rate of cardiovascular complications in coronary patients. Insights into the cardioprotective effect of certain nutriments. JACC 28, 1103-1108.

22. de Lorgeril M, Salen P \& Caillat-Vallet E (1997) Control of bias in dietary trial to prevent coronary recurrences. The Lyon Diet Heart Study. Eur J Clin Nutr 51, 116-122.

23. The Women's Healthy Eating and Living Randomized Trial (2007) Influence of a diet very high in vegetables, fruit, and fiber and low in fat on prognosis following treatment for breast cancer. JAMA 298, 289-298.

24. Hashim YZ, Eng M \& Gill CI (2005) Components of olive oil and chemoprevention of colorectal cancer. Nutr Rev $\mathbf{6 3}$, 3743-3786.

25. MacLean CH, Newberry SJ \& Mojica WA (2006) Effects of omega-3 fatty acids on cancer risk. A systematic review. JAMA 295, 403-415.

26. Larsson SC, Kumlin M \& Ingelman-Sundberg M (2004) Dietary long-chain n-3 fatty acids for the prevention of cancer: a review of potential mechanisms. Am J Clin Nutr 79, 935-945. 
27. Mitrou PN, Kipnis V, Thiebaut ACM et al. (2007) Mediterranean dietary pattern and prediction of all-cause mortality in a US population. Arch Intern Med 167, 2461-2468.

28. Knoops KT, de Groot L \& Kromhout D (2004) Mediterranean diet, lifestyle factors, and 10-year mortality in elderly European men and women. The HALE Project. JAMA 292, 1433-1439.

29. Kris-Etherton P, Eckel RH \& Howard BV (2001) AHA Science Advisory: Lyon Diet Heart Study. Benefits of a Mediterranean-style, National Cholesterol Education Program/American Heart Association Step I Dietary Pattern on Cardiovascular Disease. Circulation 103, 1823-1825.

30. Kastorini CM, Milionis HJ \& Esposito K (2011) The effect of Mediterranean diet on metabolic syndrome and its components a meta-analysis of 50 studies and 534,906 individuals. JACC 57, 1299-1313.

31. Mosca L, Benjamin EJ \& Berra K (2011) Effectiveness-based guidelines for the prevention of cardiovascular disease in women- 2011 update. JACC 57, 1404-1423.

32. Katan MB (2009) Omega-6 polyunsaturated fatty acids and coronary heart disease. Am J Clin Nutr 89, 1283-1284.

33. Ridker PM \& Glynn RJ (2010) The JUPITER Trial: responding to the critics. Am J Cardiol 106, 1351-1356.

34. Zeghichi S, Kallithraka S, Simopoulos AP et al. (2003) Nutritional composition of selected wild plants in the diet of Crete. World Rev Nutr Diet 91, 22-40.

35. de Lorgeril M, Salen P, Martin JL et al. (2008) Interactions of wine drinking with omega-3 fatty acids in coronary heart disease patients. A fish-like effect of moderate wine drinking. Am Heart J 155, 175-181.

36. Simopoulos A (2003) Importance of the ratio of omega-6/ omega-3 essential fatty acids: evolutionary aspects. World Rev Nutr Diet 92, 1-22.
37. Toufektsian MC, de Lorgeril M, Nagy N et al. (2008) Chronic dietary intake of plant-derived anthocyanins protects the rat heart against ischemia-reperfusion injury. J Nutr 138, 747-752.

38. Di Castelnuovo A, Costanzo S \& Bagnardi V (2006) Alcohol dosing and total mortality in men and women. An updated meta-analysis of 34 prospective studies. Arch Intern Med 166, 2437-2445.

39. de Lorgeril M, Salen P \& Martin JL (2002) Wine drinking and risks of cardiovascular complications after recent acute myocardial infarction. Circulation 106, 1465-1469.

40. Mukamal KJ, Maclure M, Muller JE et al. (2011) Prior alcohol consumption and mortality following acute myocardial infarction. JAMA 285, 1965-1970.

41. Janszky I, Ljung R \& Ahnve S (2008) Alcohol and long-term prognosis after acute myocardial infarction. The SHEEP Study. Eur Heart J 29, 45-53.

42. Guiraud A, de Lorgeril M \& Boucher F (2004) Cardioprotective effect of chronic low dose ethanol drinking: insights into the concept of ethanol preconditioning. JMCC $\mathbf{3 6}$, 561-566.

43. Albert CM, Manson JE \& Cook NR (1999) Moderate alcohol consumption and the risk of sudden cardiac death among US male physicians. Circulation 100, 944-950.

44. Zheng ZJ, Croft JB, Giles WH et al. (2001) Sudden cardiac death in the United States, 1989 to 1998. Circulation 104, 2158-2163.

45. Strandberg TE, Strandberg AY \& Salomaa VV (2007) Alcoholic beverage preference, 29-year mortality, and quality of life in men in old age. J Geront 62, 213-218.

46. Di Castelnuovo A, Rotondo S, Iacoviello L et al. (2002) Meta-analysis of wine and beer consumption in relation to vascular risk. Circulation 105, 2836-2844. 